\title{
URLC10 Peptide Vaccine
}

National Cancer Institute

\section{Source}

National Cancer Institute. URLC10 Peptide Vaccine. NCI Thesaurus. Code C74087.

A cancer vaccine containing URLC10 (up-regulated lung cancer 10) epitopes with potential immunostimulatory and antineoplastic activities. Upon administration, URL peptide vaccine may stimulate a cytotoxic $T$ lymphocyte $(C T L)$ response against URLC10expressing tumor cells. Up-regulated in lung and esophageal cancers, the function of URLC10 is unknown. 